\title{
ПЛАСТИКА БОЛЬШИХ И ГИГАНТСКИХ ГРЫЖ ПИЩЕВОДНОГО ОТВЕРСТИЯ ДИАФРАГМЫ С ИСПОЛЬЗОВАНИЕМ ПОЛИПРОПИЛЕНОВЫХ СЕТЧАТЫХ ИМПЛАНТАТОВ
}

\section{PLASTY OF LARGE \\ AND GIANT ESOPHAGEAL HERNIA OF THE DIAPHRAGM WITH THE USE OF POLYPROPYLENE MESH IMPLANTS}

I. Rosenfeld

Summary. The problem of increasing the efficiency of surgical treatment of diaphragmatic hernias is solved by improving the technique of surgery and choosing the type of implants. Both issues are scattered in medical literature and surgical practice, however, there is a significant gap in relation to large and gigantic hiatal hernias, which shows a high percentage of relapses. To solve this scientific problem, an integrated approach is proposed - a minimal traumatic technique of operations and a new type of implant.

Purpose of the study. Evaluation of the effectiveness of modified alloplasty using polypropylene mesh implants in patients with a diagnosis of «hiatal hernia» of large $(10-20 \mathrm{~cm} 2)$ and gigantic (more than $20 \mathrm{~cm} 2)$ sizes.

Patients and methods. Only 89 patients with large and giant diaphragmatic hernias were operated from 2014 to 2017. An analysis was made of immediate and long-term results after surgery. The mathematical system for evaluating the results included the calculation of the statistical deviation from the average indicators and the overall result.

Results. Repeated surgery was performed in 3 patients (10.3\%): 1st patient for symptomatic anatomical relapse and functional relapse, 1st patient for symptomatic anatomical relapse, and 1st patient for esophageal stricture. The effectiveness of the proposed technique increases the result of the operation and forms a decrease in the risk of relapse to $3 \%$.

Conclusion. For large and giant hernias of the esophageal opening of the diaphragm, the modified plastic with a polypropylene mesh implant shows excellent results in the frequency of anatomical relapses. Prospects for reducing the percentage (3.4\%) of relapses are seen in improving the surgical technique for performing surgery for hiatal hernia using polypropylene mesh implants.

Keywords: large hiatal hernia, giant hiatal hernia, alloplasty, polypropylene mesh implant, surgery technique.

\author{
Розенфельд Игорь Игоревич \\ К.м.н., врач-хирург, ФГБОУВО «Тверской \\ государственный медицинский университет» \\ Министерства здравоохранения Российской Федерации \\ iiggo@mail.ru
}

Аннотация. Проблема повышения эффективности хирургического лечения диафрагмальных грыж разрешается за счет совершенствования техники операции и выбора типа имплантатов. Оба вопроса разрознено рассмотрены в медицинской литературе и хирургической практике, однако, в отношении больших и гигантских грыж пищеводного отверстия диафрагмы существует значительный пробел, что показывает высокий процент рецидивов. Для решения данной научной задачи предложен комплексный подход минимальная травматическая техника операций и новый тип имплантата.

Цель исследования. Оценка эффективности модифицированной аллопластики с использованием полипропиленовых сетчатых имплантатов у пациентов с диагнозом «грыжи пищеводного отверстия диафрагмы» большого (10-20 см2) и гигантского (более 20 см2) размера.

Пациенты и методы. Прооперировано всего 89 пациентов с большими и гигантскими диафрагмальными грыжами с 2014 по 2017 гг. Был произведен анализ непосредственных и отдаленных результатов после оперативного вмешательства. Математическая система оценки результатов включала расчет статистического отклонения от средних показателей и общий результат.

Результаты. Повторная операция была выполнена 3 пациентам (10,3\%): 1-му пациенту по поводу симптомного анатомического рецидива и функционального рецидива, 1-му пациенту по поводу симптомного анатомического рецидива, а также 1-му пациенту по поводу стриктуры пищевода. Эффективность предложенной техники повышает результат операции и формирует снижение доли риска рецидива до 3\%.

Заключение. При больших и гигантских грыжах пищеводного отверстия диафрагмы модифицированная пластика полипропиленовым сетчатым имплантатом показывает отличные по частоте анатомических рецидивов результаты. Перспективы снижения процента $(3,4 \%)$ рецидивов видятся в совершенствовании хирургической техники проведения операции при грыжах пищеводного отверстия диафрагмы с использованием полипропиленовых сетчатых имплантатов.

Ключевые слова: большие грыжи пищеводного отверстия диафрагмы, гигантские грыжи пищеводного отверстия диафрагмы, аллопластика, полипропиленовый сетчатый имплантат, техника операции. 
A ллопластка пищеводного отверстия диафрагмы выполняется по 4 основным методикам. Наиболее распространенная методика - усиление задней крурорафии, то есть подшивка сетки прямоугольной, треугольной или V-образной формы к обеим ножкам диафрагмы после их сшивания позади пищевода.

Полипропиленовые имплантаты (например, «Prolene» и «Ethicon») имеют наибольшую прочность и поэтому обеспечивают формирование плотного, надежного рубца [1]. Вторым их преимуществом является экономический фактор - низкая стоимость.

Опросы С. T. Frantzides и соавторов на 264 респондентах и 5486 операциях показали, что полипропиленовые имплантаты в своей практике применяют 25,6\% хирургов [2]. В обзоре Е. Furnee и соавторов в 26 исследованиях и 924 операциях их применили в 23,1\% случаев [3]. Обзор S. A. Antoniou и соавторов в 23 исследованиях и 1445 пациентах показал, что частота анатомических рецидивов при использовании полипропиленовых сетчатых имплантатов составила 0,0-22,7\%, в среднем - 1,9\% [4]. В проспективном рандомизированном исследовании F. A. Granderath и соавторов на 50 пациентах в группе пластики полипропиленовой сеткой и послеоперационным наблюдением в среднем 1 год анатомические рецидивы были отмечены в $8 \%$ случаев $[5,6]$. В опросе C.T. Frantzides и соавторов частота анатомических рецидивов при использовании полипропиленовых сетчатых имплантатов составила 0,8\%, что составляет 5,2\% от всех рецидивов при использовании всех видов сеток. В исследовании E. Soricelli и соавторов частота анатомических рецидивов после «onlay» аллопластики полипропиленовым имплантатом составила 1,8\%, после «inlay» аллопластики полипропиленовым имплантатом - 2,4\% [7].

Недостатком полипропиленовых имплантатов является их чрезмерная жесткость. При близком расположении к пищеводу они могут вызвать чрезмерный периэзофагеальный фиброз, что приводит к длительной, более 3 месяцев, дисфагии без признаков стриктуры пищевода, или к развитию рубцовой стриктуры пищевода, а также к аррозии пищевода с миграцией сетки в его просвет. Каждое из этих осложнений может сопровождаться инфицированием имплантата вплоть до абсцедирования.

Вторым недостатком полипропиленовых имплантатов является возможность уменьшения их в объеме на 15-20\% со временем, так называемое сморщивание сетки, которое при фиксации ее вокруг пищевода усиливает возможность указанных осложнений со стороны пищевода. Так, в обзоре S.A. Antoniou и соавторов частота дисфагии при использовании полипропилено- вых сеток составила 0,0-21,7\%, в среднем - 3,9\% [4]. В проспективном рандомизированном исследовании F. A. Granderath и соавторов частота дисфагии через 1 год после операции составила 4\% [5]. В опросе C. T. Frantzides и соавторов частота рубцовых стриктур пищевода при использовании полипропиленовых имплантатов составила 0,10\%, а аррозия пищевода -0,45\% [2].

Преимуществом использования полипропиленовых имплантатов является лучшие результаты в плане профилактики рецидива, поскольку методика наиболее биомеханически физиологическая, недостатками контакт переднего края сетки с пищеводом с развитием пищеводных осложнений, особенно, если используется жесткий полипропиленовый имплантат, а также невозможность пластики дефекта впереди пищевода.

В обзоре E. Furnee и соавторов на 924 операциях методика «inlay» использовалась в $90 \%$ случаев [3]. В опросе C.T. Frantzides и соавторов методика «inlay» при использовании полипропиленовых имплантатов была применена в 87\% пластик [2].

Другая методика является модификацией первой и заключается в «onlay» усилении крурорафии цельной сеткой квадратной или округлой формы с разрезом в виде замочной скважины вокруг пищевода или отдельно сшитых между собой полукруглых участках впереди и сзади от пищевода. Преимуществом ее является возможность пластики дефекта не только позади пищевода, но и впереди его, недостатком - большая частота пищеводных осложнений из-за циркулярного столкновения имплантата с пищеводом, что усиливается сморщиванием сетки, особенно при использовании жестких полипропиленовых имплантатов [2].

F.A.Granderath и соавторы в своей ранней работе при выборочном подходе получили минимум рецидивов. Так, из 32 пациентов со средней площадью пищеводного отверстия диафрагмы 3,9 см², которым выполняли крурорафию, был только 1 рецидив. Из 12 пациентов со средней площадью грыжевого дефекта диафрагмы 7,2 см², которым выполнена «onlay» аллопластика полипропиленовым имплантатом, рецидивов не было. Из 6 пациентов со средней площадью пищеводного отверстия диафрагмы 8,5 см², которым выполнили «onlay» аллопластику композитным политетрафторэтиленовным имплантатом, рецидивов не отмечалось [6].

Методика пластики полипропиленовым имплантатом предусматривает использование стандартной или облегченной сетки с фиксацией ее поверх сшитых ножек диафрагмы $[8,9,10]$. При этом формируется грубая рубцовая ткань, происходит значительное сморщивание сетки, сохраняется контакт имплантата с пищеводом. 


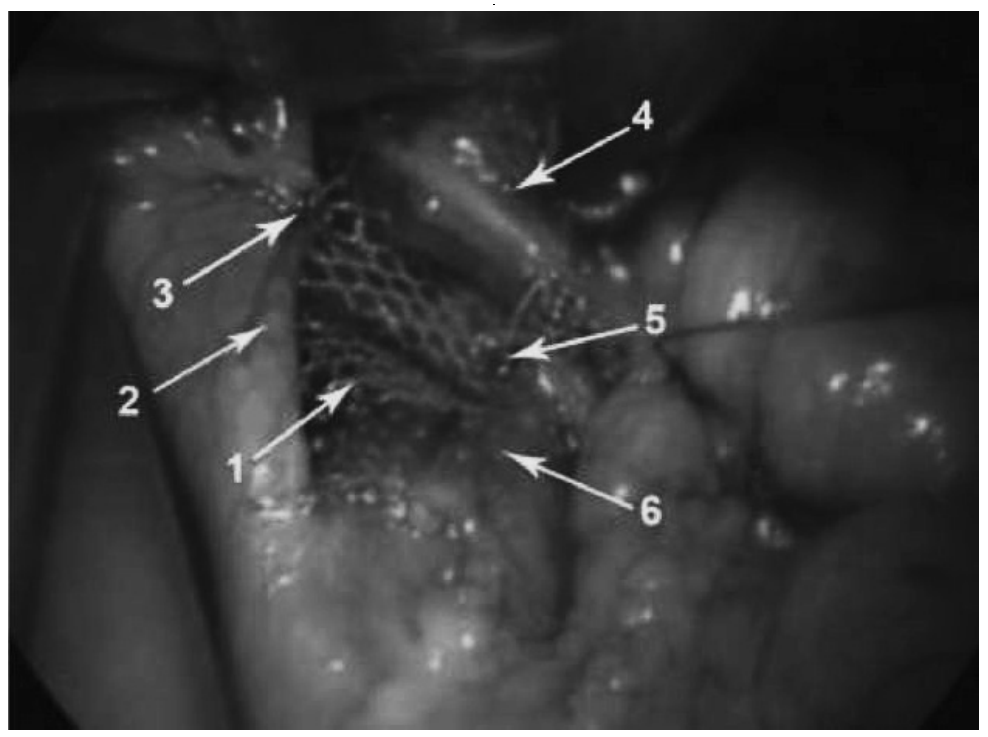

Рис. 1. Фиксация имплантата к ножкам диафрагмы:1 - сетчатый имплантат; 2 - правая ножка диафрагмы; 3 - шов, фиксирующий имплантат правой ножки диафрагмы; 4 - пищевод; 5 - шов, фиксирующий имплантат до левой ножки диафрагмы; 6 - левая ножка диафрагмы.

\section{Цель исслеАования}

Оценка эффективности модифицированной аллопластики с использованием полипропиленовых сетчатых имплантатов у пациентов с диагнозом «грыжи пищеводного отверстия диафрагмы» большого (10-20 см²) и гигантского (более $20 \mathrm{~cm}^{2}$ ) размера.

\section{Пациенты и метолы}

На диагностическом этапе исследования осуществлялась оценка степени интенсивности боли по визуальной аналоговой шкале «VAS», средний балл качества жизни по опроснику «GERD — HRQL», фиброэзофагогастродуоденоскопия, рентгеноскопия пищевода и желудка, суточный внутренний пищеводный $\mathrm{pH}$ - мониторинг.

Модифицированная аллопластика была выполнена у 89 пациентов (основная группа) (51 женщина, 38 мужчин) с диагнозом «грыжи пищеводного отверстия

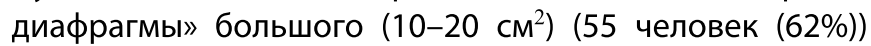
и гигантского (более $20 \mathrm{~cm}^{2}$ ) размера (34 пациента (38\%)), которые были прооперированные на базе хирургических отделений стационаров города Твери с 2014 по 2017 гг. разными бригадами врачей.

Выполнялась оценка вертикального и горизонтального размеров пищеводного отверстия диафрагмы, а также расчёты площади грыжевого дефекта. Вертикальный размер определялся между максимально верхней и максимально нижней точками пищеводного отверстия диафрагмы в месте соединения ножек. Горизонтальный размер - как расстояние между максимально удаленными точками правой и левой ножек пищеводного отверстия диафрагмы. Расчёт площади грыжевого дефекта осуществлялся по упрощённой формуле Granderath F.A. И соавторов: «S = hxv/2», где $S$ - площадь грыжевого дефекта пищеводного отверстия диафрагмы $\left(\mathrm{cm}^{2}\right), \mathrm{h}-$ горизонтальный размер (см), v - вертикальный размер (сM).

Критериями включения в применении данного способа оперативного лечения были возраст старше 18 лет, информированное согласие пациента. Из исследования исключались пациенты, сопутствующими злокачественными заболеваниями, декомпенсированной сердечно-сосудистой, дыхательной, почечной или печеночной недостаточностью, с противопоказаниями к проведению общей анестезии. Средняя продолжительность операции составила 60,6 \pm 9,2 (50-85) минут.

Лапароскопические операции выполнялись под интубационной многокомпонентной анестезией с помощью лапароскопического оборудования и инструментов фирмы «Storz». Больной находился в положении Фаулера (15-20), хирург и ассистент стояли справа от больного, а ассистент с видеокамерой - слева от больного (американская позиция). Операцию выполняли с давлением пневмоперитонеума 12-15 мм рт. ст. через 4 троакара. Конверсии отсутствовали.

Распространённый и близкий к предложенному техническому решению способ пластики пищеводного отверстия диафрагмы полипропиленовым сетчатым 


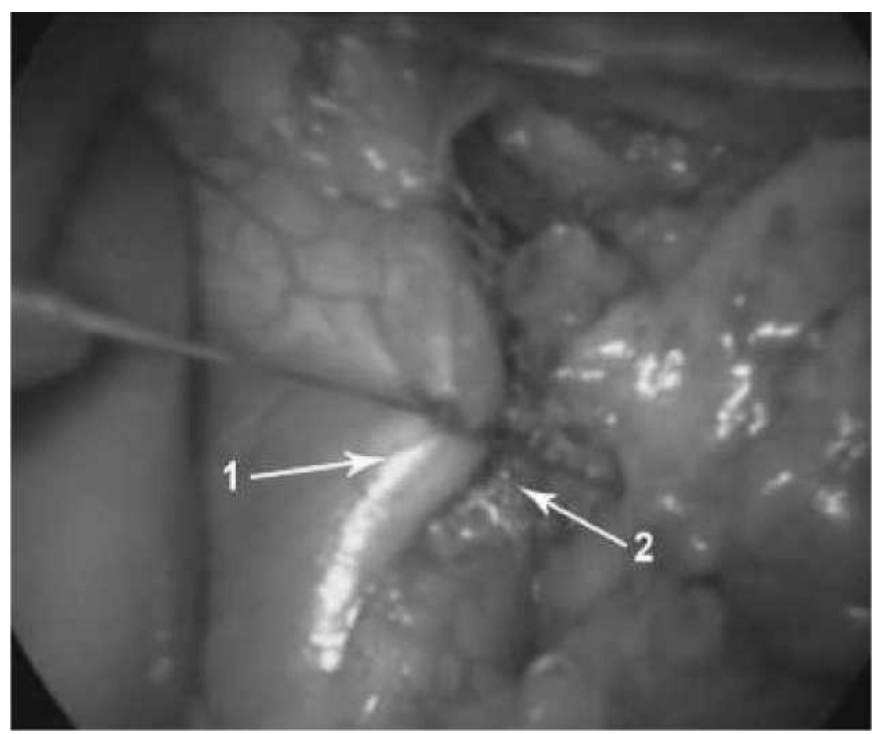

Рис. 2. Сшивание ножек с закрытием имплантата: 1 - правая ножка диафрагмы; 2 - левая ножка диафрагмы.

имплантатом - усиление задней диафрагмокрурорафии, а именно, подшивка имплантата узловыми швами к правой и левой ножкам диафрагмы после их сшивания позади абдоминального отдела пищевода. При этом верхний край конструкции может травмировать пищевод с развитием рубцовой стриктуры или деформации. Особенно это касается пластики больших и гигантских диафрагмальных грыж. При одновременной фиксации имплантата вместе с диафрагмальными ножками сетка может гофрироваться, что будет приводить к избыточному разрастанию грубой фиброзной ткани в зоне установки конструкции.

Для выполнения модифицированной пластики по разработанной методике сначала мобилизуются ножки диафрагмы и мигрировавшая часть желудка с удалением грыжевого мешка и созданием позадипищеводного туннеля, мобилизация дна желудка путем частичного рассечения желудочно-селезеночной связки и мобилизация пищевода с устранением его укорочения путем параэзофагеальной медиастинальной диссекции. Потом выполняется собственно пластика. Сначала края сетчатого полипропиленового имплантата в форме равнобедренного треугольника с длиной сторон по 4 см и длиной основания 3 см фиксируются к каждой из ножек диафрагмы позади пищевода 2-3 узловыми швами с помощью нерассасывающегося шовного материала (рис. 1).

При этом верхний край имплантата в виде основания треугольника не доходит до пищевода, полностью поднятого кверху на 1 см, для исключения контакта. Таким

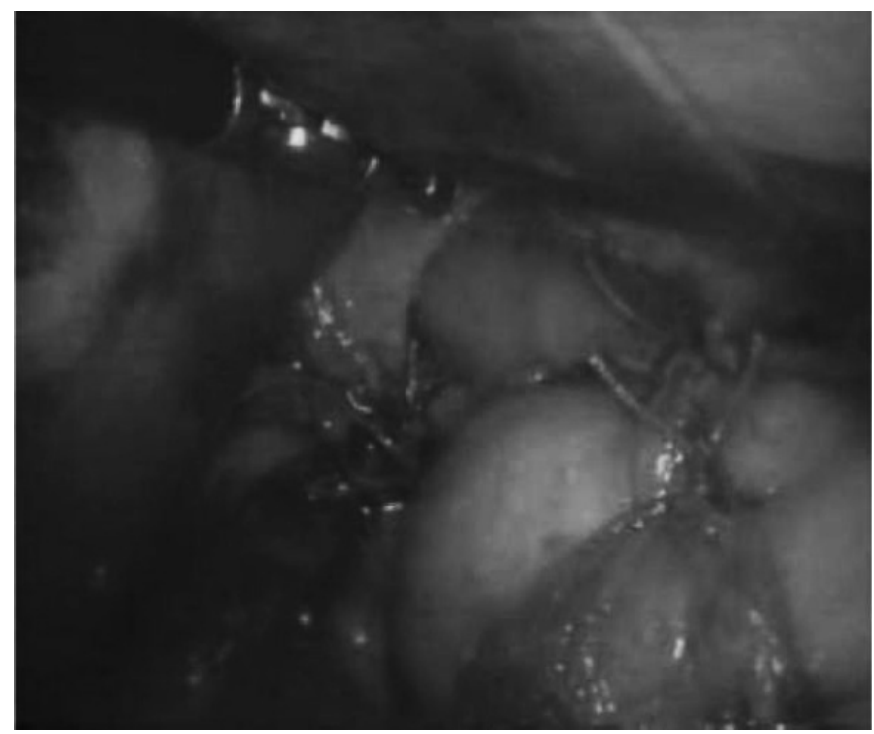

Рис. 3. Фундопликационная манжетка по Ниссену.

образом, создается «первый пласт». Потом 2-3 узловыми швами ножки диафрагмы сшиваются между собой, что приводит к полному закрытию имплантата (рис. 2).

При этом верхний шов не доходит до пищевода, полностью поднятого на 0,5-1,0 см кверху в сторону головного конца. Таким образом, создается «второй пласт». Пластика выполняется при введенном в желудок калибровочном зонде диаметром 30 мм. Потом выполняется фундопликация по Ниссену с длиной манжетки 3 см, а также шовным материалом, который не рассасывается, с использованием калибровочного зонда (рис. 3).

Таким образом, данный имплантат закрывается ножками диафрагмы, которые препятствуют его контакту с пищеводом, что не вызывает «пилящего» эффекта при дыхательных движениях диафрагмы.

Отдельно была проанализирована дополнительная группа пациентов (75 человек: 45 женщин и 30 мужчин), которым была выполнена «onlay» пластика пропиленовым имплантатом.

Базовая, или описательная, статистика включала дискретное изучения основных характеристик вариационных рядов: среднее арифметическое, стандартное отклонение, минимальное значение, максимальное значение. Данные представлены в виде среднего значения (М) и стандартной ошибки средней $(\mathrm{m})$. Значимость различий оценивали по критерию Вилкоксона, считая статистически достоверным значение $p<0,05$. 
Таблица. Отдаленные результаты

Table. Long-term results

\begin{tabular}{|l|l|}
\hline Анатомический рецидив всех видов & $7(24,1 \%)$ \\
\hline Симптомный анатомический рецидив и функциональный рецидив & $2(6,9 \%)$ \\
\hline Симптомный анатомический рецидив & $3(10,3 \%)$ \\
\hline Бессимптомный анатомический рецидив и функциональный рецидив & $1(3,4 \%)$ \\
\hline Бессимптомный анатомический рецидив & $1(3,4 \%)$ \\
\hline Функциональный рецидив всех видов & $1(3,4 \%)$ \\
\hline Симптомный функциональный рецидив & $1(3,4 \%)$ \\
\hline Бессимптомный функциональный рецидив & 0 \\
\hline Рефлюкс - эзофагит & $4(13,8 \%)$ \\
\hline Индекс «Dе Мееstег» & $31,5 \pm 53,1(4,1-187,4)$ \\
\hline Длительная функциональная дисфагия & $6(20,7 \%)$ \\
\hline Стриктуры & $1(3,4 \%)$ \\
\hline Повторные операции & $3(10,3 \%)$ \\
\hline
\end{tabular}

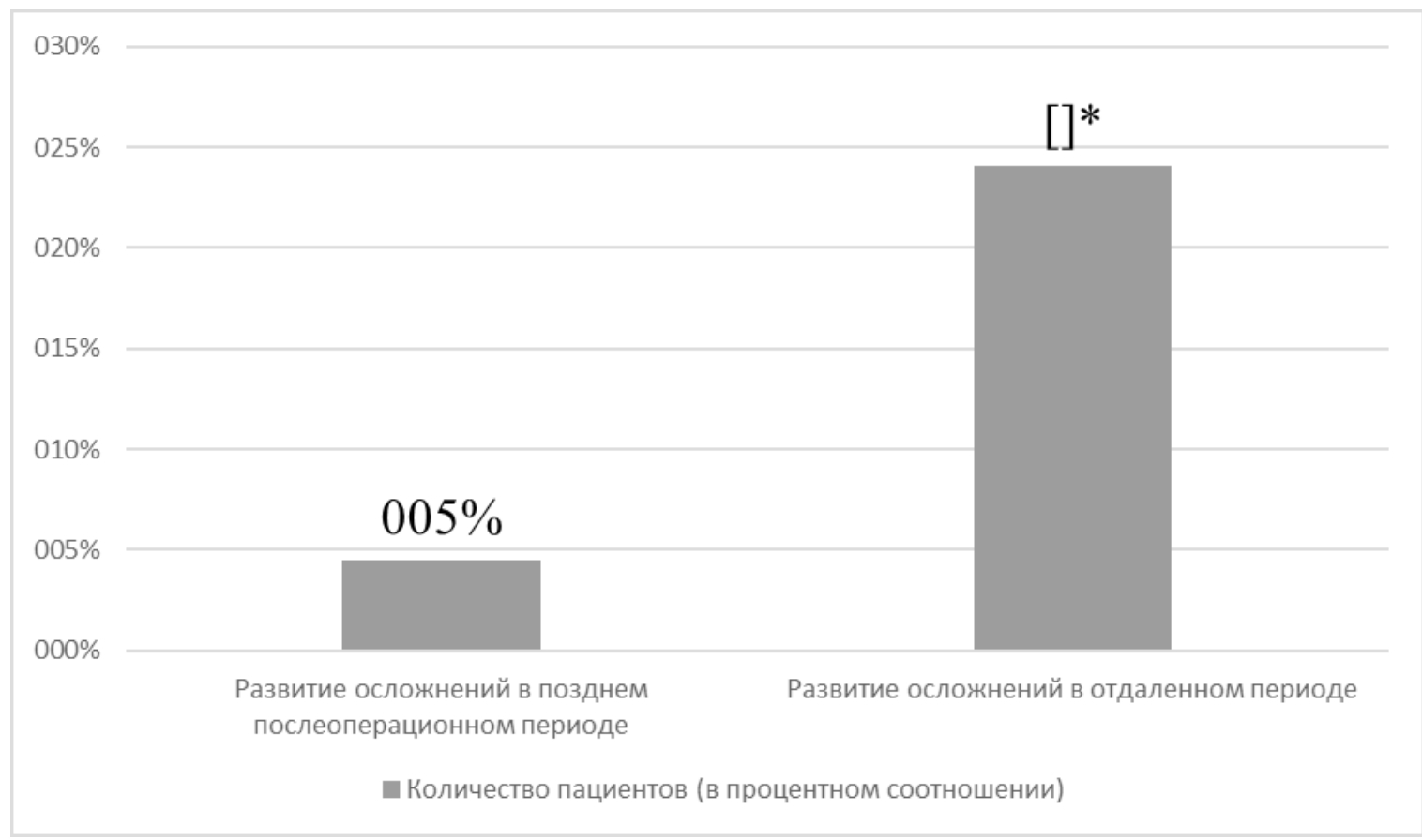

Рис. 4. Сравнительная характеристика непосредственных и отдаленных результатов исследования в плане развития осложнений (в процентном соотношении) у пациентов основной группы.

Примечание:

*p<0,05- достоверность различия признака между группами.

\section{Результаты исслеАования}

\section{Непосредственные результаты}

Интраоперационных осложнений не было. Поздние послеоперационные осложнения имели место у 4 (4,5\%) пациентов: 3 случая ателектазов с лихорадкой, которые относились к II степени осложнений по классификации «Clavien - Dindo», а также 1 случай поддиафрагмальной гематомы, относящейся к IIIA степени по классификации «Clavien - Dindo», и которая требовала пункционного дренирования. Средний послеоперационный койко-день составил 5,5 $\pm 1,5$ (2-10) дней.

\section{Отдаленные результаты}

Из 89 пациентов анатомические рецидивы всех видов были выявлены у 7 (24,1\%). Они были распределе- 


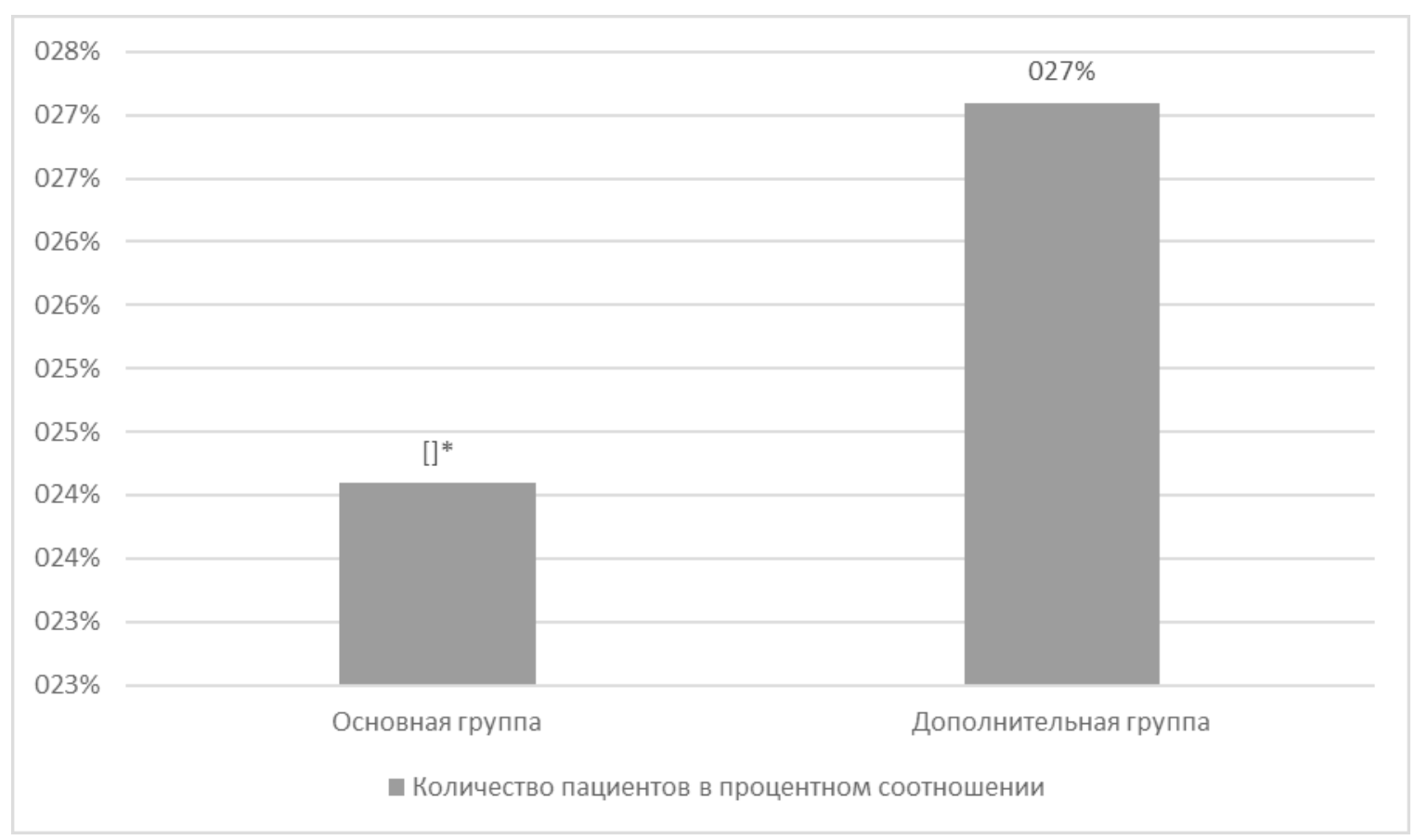

Рис. 5. Сравнительная характеристика данных отдаленных результатов исследования основной и дополнительной групп.

Примечание:

*p<0,05- достоверность различия признака между группами

ны следующим образом: симптомный анатомический рецидив и функциональный рецидив были выявлены у 2 (6,9\%), симптомный анатомический рецидив - у 3 (10,3\%), бессимптомный анатомический рецидив и функциональный рецидив - у 1 (3,4\%), бессимптомный анатомический рецидив - у 1 пациента $(3,4 \%)$. Симптомный функциональный рецидив выявлен у 1 (3,4\%), бессимптомные функциональные рецидивы отсутствовали, рефлюкс - эзофагит был выявлен у 4 (13,8\%) (таблица).

Средний индекс «De Meester» (показатель экспозиции соляной кислоты в пищеводе при норме менее 14,72) у 24 пациентов составил 31,5 $\pm 53,1(4,1-187,4)$. Длительная функциональная дисфагия имела место у 6 (20,7\%). Стриктура диафрагмы имела место в 1 случае $(3,4 \%)$ и развилась спустя 3 месяца после операции. Повторная пластика грыжи пищеводного отверстия диафрагмы была выполнена 3 (10,3\%): 1 пациенту по поводу симптомного анатомического рецидива и функционального рецидива, 1 пациенту по поводу симптомного анатомического рецидива, а также 1 пациенту по поводу стриктуры.

Сравнительная характеристика непосредственных и отдаленных результатов исследования в плане развития осложнений (в процентном соотношении) представлена на рисунке 4.
Основными причинами развития осложнений в отдаленном периоде являются анатомические рецидивы всех видов.

Сравнительная характеристика данных отдаленных результатов исследования основной и дополнительной групп представлена на рисунке 5 .

Эффективность предложенной конструкции имплантата достоверно $(p<0,05)$ повышает результат операции и формирует снижение доли риска рецидива по результатам отдаленных результатов в сравнении с «onlay» пластикой пропиленовым имплантатом до 3\%.

\section{Обсужление результатов}

Грыжи пищеводного отверстия диафрагмы представляют собой важную проблему в современной медицинской практике в связи с тем, что оказывают влияние на показатели физического здоровья, способствуя снижению качества жизни. Это объясняется достаточно частым развитием осложнений, среди которых первостепенное значение играет гастроэзофагеальная рефлюксная болезнь (ГЭРБ). В работе Оскреткова В.И. и соавт. было продемонстрировано, что выполнение видеолапароскопической протезирующей диафрагмохиатопластики при грыжах ПОД с расширением более 5 см способствует предот- 
вращению рецидива заболевания [11]. Важное значение было уделено вопросу материала, который бы обеспечивал антиспаечный барьер по отношению к органам брюшной полости. В нашей работе также была выбрана методика с применением закрытия имплантата ножками диафрагмы с целью отграничения его контакта с пищеводом. Другие исследователи полагают, что использование лапароскопического хирургического лечения диафрагмальной грыжи большого и гигантского размеров приводят к частому развитию рецидивов в течение 5-летнего периода [12]. В нашей работе также отмечен факт того, что развитие осложнений в отдаленном периоде превышало их количество в позднем послеоперационном периоде в основном за счет анатомических рецидивов всех видов. В литературе последних лет встречаются работы, в которых чаще применяются оригинальные методики хирургического лечения больших и гигантских грыж ПОД (например, Черкасов Д.М. и соавторы использовали сетчатый имплантат, который устанавливали и фиксировали в заднее средостение над диафрагмой) [13]. По наблюде- ниям авторов, в отдаленном периоде хорошие результаты были зафиксированы у большинства пациентов (у 23 из 25). В нашей работе при проведении сравнения предложенной методики в сравнении с «onlay» пластикой пропиленовым имплантатом также зафиксировано улучшение результатов со снижением количества развития осложнений у основной группы в отдаленном периоде по сравнению с дополнительной.

\section{Зак^Ючение}

При больших и гигантских грыжах пищеводного отверстия диафрагмы модифицированная пластика полипропиленовым сетчатым имплантатом показывает неплохие по частоте анатомических рецидивов результаты. Перспективы снижения процента рецидивов видятся в совершенствовании хирургической техники проведения операции при грыжах пищеводного отверстия диафрагмы с использованием полипропиленовых сетчатых имплантатов.

\section{ЛИТЕРАТУРА}

1. Можаровский В.В., Цыганов А. А., Можаровский К. В., Тарасов А. А. Оценка эффективности хирургического лечения гастроэзофагеальной рефлюксной болезни в сочетании с грыжей пищеводного отверстия диафрагмы. Хирургия. Журнал им. Н. И. Пирогова. 2017;6:28-32.

2. Frantzides C.T., Carlson M. A., Loizides S. et al. Hiatal hernia repair with mesh: a survey of SAGES members. Surgical endoscopy. 2016;24(5):1017-1024. D0I: 10.1007/ s00464-009-0718-6

3. Furnée E., Hazebroek E. Mesh in laparoscopic large hiatal hernia repair: a systematic review of the literature. Surgical endoscopy. 2015;27(11):3998-4008. D0I: 10.1007/s00464-013-3036-y

4. Antoniou S. A., Koch 0. 0., Antoniou G. A. et al. Mesh-reinforced hiatal hernia repair: a review on the effect on postoperative dysphagia and recurrence. Langenbeck's archives of surgery. 2015;397(1):19-27. D0l: 10.1007/s00423-011-0829-0

5. Granderath F. A., Schweiger U. M., Kamolz T. et al. Laparoscopic Nissen fundoplication with prosthetic hiatal closure reduces postoperative intrathoracic wrap herniation: preliminary results of a prospective randomized functional and clinical study. Archives of surgery. 2015;140(1):40-48. D0I: 10.1001/archsurg.140.1.40

6. Granderath F. A., Schweiger U. M., Pointner R. Laparoscopic antireflux surgery: Tailoring the hiatal closure to the size of hiatal surface area. Surgical endoscopy. 2017;21(4):542-548. D0I: 10.1007/s00464-006-9041-7

7. Soricelli E., Basso N., Genco A. et al. Long-term results of hiatal hernia mesh repair and antireflux laparoscopic surgery. Surgical endoscopy. 2016;23(11):2499-2504. DOI: 10.1007/s00464-009-0425-3

8. Грубник В.В., Малиновский А. В. Критические аспекты лапароскопической хирургии гастроэзофагеальной рефлюксной болезни и грыж пищеводного отверстия диафрагмы. Одесса: ВМВ-типография. 2015:106.

9. Велигоцкий М.М., Горбулич А. В., Комарчук В. В. Профилактика неблагоприятных результатов лапароскопической коррекции антирефлюксной функции кардии при грыжах пищеводного отверстия диафрагмы и ахалазии пищевода. Одесский медицинский журнал. 2015;149(3):66-69.

10. Ратчик В.М., Пролом Н. В., Шевченко Б. Ф., Бабий А. М., Буренко А. Н. Опыт хирургического лечения грыж пищеводного отверстия диафрагмы лапароскопическим доступом. Гастроэнтерология. 2017;51(3):13-17.

11. Оскретков В.И., Ганков В. А., Гурьянов А. А., Андреасян А. Р., Федоров В. В., Шестаков Д. Ю. Видеолапароскопическая протезирующая диафрагмохиатопластика при аксиальных грыжах пищеводного отверстия диафрагмы с признаками ГЭРБ. Медицина и образование в Сибири. 2014;4:10-11.

12. Тимербулатов М.В., Сендерович Е. И., Гришина Е. Е., Гарифуллин Б. М., Гимаев Э. Ф., Казаков Н. М., Ибрагимов Р. К. Отдаленные результаты эндохирургического лечения параэзофагеальных грыж пищеводного отверстия диафрагмы. Креативная хирургия и онкология. 2017; 7(4):4-10.

13. Черкасов Д.М., Черкасов М. Ф., Татьянченко В. К., Старцев Ю. М., Меликова С. Г., Галашокян К. М. Хирургическая тактика при больших и гигантских грыжах пищеводного отверстия диафрагмы. Вестник хирургии имени И. И. Грекова. 2018; 177(4):63-66. 